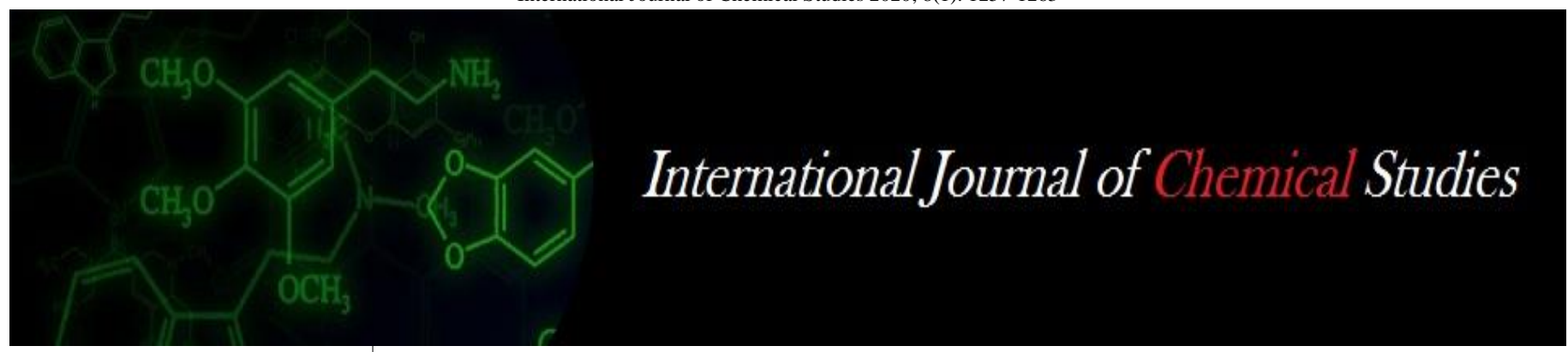

P-ISSN: 2349-8528

E-ISSN: 2321-4902

IJCS 2020; 8(1): 1257-1263

(C) 2020 IJCS

Received: 24-11-2019

Accepted: 28-12-2019

\section{SS Sawant}

Department of Agricultural Botany, College of Agriculture, Dapoli, Dr. Balasaheb Sawant Konkan Krishi Vidyapeeth, Dapoli, Ratnagiri, Maharashtra, India

\section{SS Sawant}

Department of Agricultural Botany, College of Agriculture, Dapoli, Dr. Balasaheb Sawant Konkan Krishi Vidyapeeth, Dapoli, Ratnagiri, Maharashtra, India

\section{SS Sawant}

Department of Agricultural Botany, College of Agriculture, Dapoli, Dr. Balasaheb Sawant Konkan Krishi Vidyapeeth, Dapoli, Ratnagiri, Maharashtra, India

\section{SS Sawant}

Department of Agricultural Botany, College of Agriculture, Dapoli, Dr. Balasaheb Sawant Konkan Krishi Vidyapeeth, Dapoli, Ratnagiri, Maharashtra, India

\section{SS Sawant}

Department of Agricultural Botany, College of Agriculture, Dapoli, Dr. Balasaheb Sawant Konkan Krishi Vidyapeeth, Dapoli, Ratnagiri,

Maharashtra, India

\section{SS Sawant}

Department of Agricultural Botany, College of Agriculture, Dapoli, Dr. Balasaheb Sawant Konkan Krishi Vidyapeeth, Dapoli, Ratnagiri,

Maharashtra, Indi

\section{SS Sawant}

Department of Agricultural Botany, College of A griculture, Dapoli, Dr. Balasaheb Sawant Konkan Krishi Vidyapeeth, Dapoli, Ratnagiri, Maharashtra, India
Corresponding Author: SS Sawant

Department of Agricultural Botany, College of Agriculture, Dapoli, Dr. Balasaheb Sawant Konkan Krishi Vidyapeeth, Dapoli, Ratnagiri, Maharashtra, India

\section{Combining ability and gene action in cucumber (Cucumis sativus L.)}

\author{
SS Sawant, SG Bhave, VV Dalvi, JP Devmore, MM Burondkar, MH \\ Khanvilkar and BR Salvi
}

DOI: $\underline{\text { https://doi.org/10.22271/chemi.2020.v8.i1q.8425 }}$

\begin{abstract}
The experimental material for the present study comprised of $F_{1}$ population of twenty four crosses, developed by crossing four female parents viz., Sheetal, Shubhangi, Himangi, and Puna khira of cucumber with six male parents viz., AAUC-2, DC-2, AAUC-1, VRC-19, DARL-103 and Fansu local. The experiment was laid out with $24 \mathrm{~F} 1 \mathrm{~s}, 4$ females 6 males and one check Konkan kakadi in Randomized Block Design (RBD) with two replications during the kharif 2017.Observations were recorded on thirteen different quantitative characters The perusal of estimates of gca effects revealed that two female parents viz., sheetal (0.572), Puna khira (0.235) and two male parents Fansu local (0.596) and VRC-19 (0.376) exhibited significant positive gca effect in kharif 2017. This showed that these parents were good general combiner for the marketable yield vine ${ }^{-1}$. The best three hybrids with respect to marketable yield $v^{-1} e^{-1}$ based on significant positive sca were viz., Sheetal x Fansu local (0.942), Shubhangi x DC-2 (0.532), Puna khira x VRC-19 (0.484). A perusal of the data indicated that the estimates of $\sigma^{2} \mathrm{SCA}$ were higher in magnitude as compared to $\sigma^{2}$ GCA (average) both in females and males for the traits under study viz., days to first male flower appearance, days to first female flower appearance, nodal position of first female flower, days to first picking, fruit length and diameter, average fruit weight, marketable yield vine ${ }^{-1}$, harvest duration and total soluble solids. In all the traits studied, the SCA variances were higher than GCA values, indicated the role of non-additive gene action. While lower magnitude of SCA showed additive gene action. The heritability estimates from different variance components having very high heritability obtained for number of marketable fruits vine ${ }^{-1}(69.02 \%)$ and number of primary branches plant ${ }^{-1}(94.40 \%)$.
\end{abstract}

Keywords: Cucumber, $\mathrm{F}_{1}$ hybrids, GCA, SCA and yield characters

\section{Introduction}

Cucumber (Cucumis sativus L.) belongs to the family cucurbitaceae and its centre of origin is India (Renner et al., 2007) ${ }^{[17]}$. It is an important and one of the most popular fruit vegetable in Asia. Cucumber is also an ideal vegetable for its edible tender fruits, preferred as salad ingredient, pickles, desert fruit and as a cooked vegetable. Since there exist a considerable variability of this crop, it is possible to develop high yielding varieties through breeding approaches like selection or hybridization. Combining ability is one of the important and powerful tools to identifying the best combiner that may be used in crosses to exploit heterosis. It helps to know the genetic architecture of various characters that enable the breeder to design effective breeding plan for future improvement of the existing materials. The combining ability analysis gives useful information regarding the selection of parents in terms of the performance of their hybrids. Information on the relative importance of general combining ability (GCA) and specific combining ability (SCA) is of value in breeding programs for species which are amenable to the development of F1 hybrid cultivars such basic information on combining ability in cucumber would aid the breeder in developing improved hybrid cultivars (Tasdighi and Baker, 1981) ${ }^{[20]}$. The line $\mathrm{x}$ tester technique was developed by Kempthorne in $1957^{[4]}$. It is a good approach for screening the germplasm on the basis of GCA and SCA variances and effects. Line $\times$ tester analysis involving parents give the additional information about the presence or absence of epistasis, average degree of dominance, as well as distribution of dominant and recessive genes in the parents. Application of line $\times$ tester technique in a cross-pollinated crop like cucumber for this purpose may be appropriate. The estimates of gene effects and genetic variance help in understanding the 
genetic potential of the breeding material. Fruit yield being a polygenic character, many genes are involved in its inheritance. Because of the small effect of individual genes, it is apparently difficult to study their individual effects. The present investigation was undertaken to generate information for identification of good general and specific combiners of cucumber genotypes for the improvement of yield and its attribute

\section{Materials and Methods}

The experimental material for the present study comprised of $F_{1}$ population of twenty four crosses, developed by crossing four females (lines) viz., Sheetal, Shubhangi, Himangi, and Puna khira of cucumber with six males (testers) viz., AAUC2, DC-2, AAUC-1, VRC-19, DARL-103 and Fansu local were used in this experiment. All the lines used as female parents were crossed to each of the tester by hand pollination in a line $\mathrm{x}$ tester model and thus line $\mathrm{x}$ tester full-sib crossed true to type seeds was produced at theEducational Experimental Botany Farm, at Department of Agriculture Botany, College of Agriculture, Dapoli during kharif 2017.The experiment was laid out with 24 F1s, 4 females 6 males and one check Konkan kakadi in Randomized Block Design (RBD) with two replications. The unit plot size was $3.0 \mathrm{~m}$ X $6.0 \mathrm{~m}$ accommodating 10 plants in each plot with spacing of $3.0 \mathrm{~m} \mathrm{X} 0.60 \mathrm{~m}$. All the recommended agronomic practices were adopted to raise a good crop. Data on 13 quantitative characters: viz. Days to first male flower appearance, days to first female flower appearance, nodal position of first female flower, days to first picking, fruit length $(\mathrm{cm})$, fruit diameter $(\mathrm{cm})$, average fruit weight $(\mathrm{g})$, number of marketable fruits vine ${ }^{-1}$, marketable yield vine ${ }^{-1}$ $(\mathrm{kg})$, harvest duration, number of primary branches plant ${ }^{-1}$, vine length $(\mathrm{m})$, total soluble solids $\left({ }^{0}\right.$ Brix) were recorded. The collected data were statistically analyzed. Analysis of variance for each of the character was performed. The data for each character was analyzed by using standard statistical procedure (Panse and Sukhatme, 1985) ${ }^{[12]}$.

\section{Results and Discussion}

\section{Analysis of variance for combining ability}

Various genetic variances were estimated from the analysis (Table 1) of variance for combining ability for thirteen characters in each season as suggested by Kempthorne (1957) [4]. The analysis of variances indicated that variance due to females and females $\times$ males was significant fordays to first male flower appearance. Regarding days to first female flower appearance variance due to females $\times$ males interaction was found to be highly significant. In case of days to first picking combining ability variances in females as well as in females $\times$ males were highly significant.

Table 1: Analysis of variance for combining ability for different characters in cucumber

\begin{tabular}{|c|c|c|c|c|c|c|c|c|c|c|}
\hline $\begin{array}{c}\text { Sr. } \\
\text { No. }\end{array}$ & $\begin{array}{c}\text { Source of } \\
\text { variance }\end{array}$ & D.F. & $\begin{array}{c}\text { Days to first male } \\
\text { flower } \\
\text { appearance }\end{array}$ & $\begin{array}{c}\text { Days to first } \\
\text { female flower } \\
\text { appearance }\end{array}$ & $\begin{array}{c}\text { Nodal position } \\
\text { of first female } \\
\text { flower }\end{array}$ & $\begin{array}{c}\text { Days to } \\
\text { first } \\
\text { picking }\end{array}$ & $\begin{array}{c}\text { Fruit } \\
\text { length } \\
\text { (cm) }\end{array}$ & $\begin{array}{c}\text { Fruit } \\
\text { diameter } \\
\text { (cm) }\end{array}$ & $\begin{array}{c}\text { Average fruit } \\
\text { weight (g) }\end{array}$ & $\begin{array}{c}\text { Number of } \\
\text { marketable } \\
\text { fruits vine }\end{array}$ \\
\hline 1 & Females & 3 & $106.909^{*}$ & 25.916 & 1889 & 18.62 & 0.553 & 0.16 & 183.85 & 0.49 \\
\hline 2 & Males & 5 & 34.83 & 20.033 & 4.133 & $11.97 * *$ & 0.888 & $2.36^{* *}$ & 379.00 & $66.383^{* *}$ \\
\hline 3 & $\begin{array}{c}\text { Females } \times \\
\text { Males }\end{array}$ & 15 & $27.326^{* *}$ & $14.533^{* *}$ & $3.0889^{* *}$ & 3.15 & $1.823^{* *}$ & 0.272 & 971.764 & 13.156 \\
\hline 4 & Error & 23 & 1.62 & 1.62 & 0.69 & 18.62 & 0.59 & 0.323 & $557.537^{* *}$ & $6.053^{* *}$ \\
\hline
\end{tabular}

Table 1: Cont.

\begin{tabular}{|c|c|c|c|c|c|c|c|}
\hline $\begin{array}{c}\text { Sr. } \\
\text { No. }\end{array}$ & $\begin{array}{c}\text { Source of } \\
\text { Variance }\end{array}$ & D.F. & $\begin{array}{c}\text { Marketable yield vine } \\
(\mathbf{k g})\end{array}$ & $\begin{array}{c}\text { Harvest } \\
\text { duration }\end{array}$ & $\begin{array}{c}\text { Number of primary branches } \\
\text { plant }^{-1}\end{array}$ & $\begin{array}{c}\text { Vine length } \\
(\mathbf{m})\end{array}$ & $\begin{array}{c}\text { Total soluble solids } \\
(\mathbf{0} \text { Brix })\end{array}$ \\
\hline 1 & Females & 3 & 0.082 & 11.13 & 0.04 & 0.29 & 0.009 \\
\hline 2 & Males & 5 & $2.845^{*}$ & 48.055 & $4.487^{* *}$ & 0.734 & 0.905 \\
\hline 3 & Females $\times$ Males & 15 & 1.465 & 48.933 & $0.156^{*}$ & 0.196 & 0.288 \\
\hline 4 & Error & 23 & $0.609^{* *}$ & 19.855 & 0.054 & 0.334 & 0.483 \\
\hline
\end{tabular}

$*$,** significant at 5 and 1percent probability level respectively

For the fruit length estimates of variances due females and males for combining ability were found to be non-significant whereas estimates of gca variances due females $\mathrm{x}$ males were significant. For average fruit weight the mean squares due to females $\mathrm{x}$ males were significant. Number of marketable fruits vine ${ }^{-1}$ showed that general combining ability variance due to females and females $\times$ males were significant.

In case of marketable yield vine ${ }^{-1}$ the mean squares due to females and females $\times$ males were found to be significant to were highly significant while, variances for males were non- significant. The variance due to females $\times$ males was non significant.

The mean square for number of primary branches plant $^{-1}$ due to females and females $\mathrm{x}$ males. Total soluble solid exhibits non - significant results in all interaction.

\section{Studies on combining ability effects}

The estimates of general combining ability (gca) effects (Table 2) of parents and specific combining ability (sca) effects of hybrid for thirteen characters are presented in (Table 3).

Table 2: General combining ability effects for different characters in cucumber

\begin{tabular}{|c|c|c|c|c|c|c|c|c|c|}
\hline $\begin{array}{c}\text { Sr. } \\
\text { No. }\end{array}$ & Female parents & $\begin{array}{c}\text { Days to first } \\
\text { male flower } \\
\text { appearance }\end{array}$ & $\begin{array}{c}\text { Days to first } \\
\text { female flower } \\
\text { appearance }\end{array}$ & $\begin{array}{c}\text { Nodal position } \\
\text { of first female } \\
\text { flower }\end{array}$ & $\begin{array}{c}\text { Days to } \\
\text { first } \\
\text { picking }\end{array}$ & $\begin{array}{c}\text { Fruit } \\
\text { length } \\
\text { (cm) }\end{array}$ & $\begin{array}{c}\text { Fruit } \\
\text { diameter } \\
\text { (cm) }\end{array}$ & $\begin{array}{c}\text { Average } \\
\text { fruit weight } \\
\text { (g) }\end{array}$ & $\begin{array}{c}\text { Number of } \\
\text { marketable } \\
\text { fruits vine }\end{array}$ \\
\hline 1 & Sheetal & $2.021^{* *}$ & 0.208 & $0.417^{*}$ & $-0.979^{* *}$ & 0.067 & $-0.541^{* *}$ & 3.552 & $2.700^{* *}$ \\
\hline 2 & Shubhangi & 0.688 & $-0.042^{* *}$ & $-0.250^{* *}$ & $-0.146^{* *}$ & $0.263^{*}$ & 0.003 & 4.444 & $-2.342^{* *}$ \\
\hline 3 & Himangi & $-4.396^{* *}$ & $-1.875^{* *}$ & $0.250^{*}$ & $-1.813^{* *}$ & $-0.091^{* *}$ & $-0.009^{* *}$ & $-0.098^{* *}$ & $-1.550^{* *}$ \\
\hline
\end{tabular}




\begin{tabular}{|c|c|c|c|c|c|c|c|c|c|}
\hline 4 & Punakhira & $1.688^{* *}$ & $1.708^{* *}$ & $-0.417^{* *}$ & $2.938^{* *}$ & $-0.238^{* *}$ & $0.548^{* *}$ & $-7.898^{* *}$ & $1.192^{* *}$ \\
\hline $\begin{array}{c}\text { No. of female parents } \\
\text { showed (-ve) significant } \\
\text { gca effects }\end{array}$ & 01 & 02 & 02 & 03 & 01 & 01 & - \\
\hline & SE(gi) + & 0.60 & 0.58 & 0.33 & 0.68 & 0.30 & 0.16 & 5.82 & 0.25 \\
\hline & Males parents & & & & & & & & \\
\hline 5 & AAUC-2 & $1.188^{*}$ & 0.292 & 0.292 & $-0.271^{* *}$ & 0.215 & $-0.171^{* *}$ & 8.610 & $0.692^{* *}$ \\
\hline 6 & DC-2 & $-2.188^{* *}$ & $-1.083^{* *}$ & $-0.208^{* *}$ & $-1.396^{* *}$ & 0.056 & $-0.203^{* *}$ & 10.523 & $-1.646^{* *}$ \\
\hline 7 & AAUC-1 & $-2.688^{* *}$ & $-1.708^{* *}$ & 0.292 & $-1.771^{* *}$ & $-0.237^{* *}$ & $-0.086^{* *}$ & $-16.865^{* *}$ & $-1.333^{* *}$ \\
\hline 8 & VRC-19 & 1.063 & $1.917^{* *}$ & $-0.333^{* *}$ & $2.229^{*}$ & 0.182 & $0.084^{*}$ & 4.123 & $0.817^{* *}$ \\
\hline 9 & DARL-103 & $-0.063^{* *}$ & $-1.208^{* *}$ & $-1.083^{* *}$ & $-0.021^{* *}$ & $0.336^{*}$ & $0.274^{* *}$ & $-10.290^{* *}$ & $-0.133^{* *}$ \\
\hline 10 & Fansu local & $2.688^{* *}$ & $1.792^{* *}$ & $1.042^{* *}$ & 1.229 & $-0.552^{* *}$ & $0.102^{*}$ & 3.898 & $1.604^{* *}$ \\
\hline $\begin{array}{c}\text { No. of female parents } \\
\text { showed (-ve) significant } \\
\text { gca effects }\end{array}$ & 03 & 01 & 03 & 03 & 01 & 03 & - & 03 \\
\hline
\end{tabular}

Table 2: cont.

\begin{tabular}{|c|c|c|c|c|c|c|}
\hline Sr. No. & Female parents & $\begin{array}{c}\text { Marketable yield } \\
\text { vine }^{-1}(\mathrm{~kg})\end{array}$ & $\begin{array}{r}\text { Harvest } \\
\text { duration }\end{array}$ & $\begin{array}{c}\text { Number of primary } \\
\text { branches plant }^{-1}\end{array}$ & \begin{tabular}{|c|}
$\begin{array}{c}\text { Vine length } \\
(\mathrm{m})\end{array}$ \\
\end{tabular} & \begin{tabular}{|c|}
$\begin{array}{c}\text { Total soluble solids } \\
\left({ }^{0} \mathrm{Brix}\right)\end{array}$ \\
\end{tabular} \\
\hline 1 & Sheetal & $0.572 * *$ & 2.667 & $0.502 * *$ & $-0.324 * *$ & $0.263^{* *}$ \\
\hline 2 & Shubhangi & $-0.360 * *$ & 0.250 & $-0.406^{* *}$ & $-0.041 * *$ & $-0.029 * *$ \\
\hline 3 & Himangi & $-0.447 * *$ & $-2.000 * *$ & $-0.640 * *$ & 0.111 & $0.138 * *$ \\
\hline 4 & Punakhira & $0.235^{* *}$ & $-0.917 * *$ & $0.544 * *$ & 0.254 & $-0.371 * *$ \\
\hline \multicolumn{2}{|c|}{$\begin{array}{l}\text { No. of female parents showed (-ve) } \\
\text { significant gca effects }\end{array}$} & 02 & - & 02 & - & 02 \\
\hline \multicolumn{2}{|c|}{$\mathrm{SE}(\mathrm{gi}) \pm$} & 0.13 & 1.25 & 0.08 & 0.52 & 0.07 \\
\hline & Males parents & & & & & \\
\hline 5 & AAUC-2 & $-0.017 * *$ & 2.792 & $0.152 * *$ & $-0.131 * *$ & $0.317 * *$ \\
\hline 6 & DC-2 & $-0.258 * *$ & 2.417 & $0.077 * *$ & 0.187 & $-0.121 * *$ \\
\hline 7 & AAUC-1 & $-0.583 * *$ & $-2.833 * *$ & 0.090 ** & 0.114 & $-0.196 * *$ \\
\hline 8 & VRC-19 & $0.376 * *$ & $-2.708 * *$ & $0.027 *$ & 0.042 & $-0.133 * *$ \\
\hline 9 & DARL-103 & $-0.114 * *$ & $-0.708 * *$ & $-0.173 * *$ & $-0.236 * *$ & $0.079 * *$ \\
\hline 10 & Fansu local & $0.596 * *$ & 1.042 & $-0.173 * *$ & 0.024 & $0.054 * *$ \\
\hline \multicolumn{2}{|c|}{$\begin{array}{l}\text { No. of female parents showed (-ve) } \\
\text { significant gca effects }\end{array}$} & 02 & - & 04 & - & 03 \\
\hline \multicolumn{2}{|c|}{$\mathrm{SE}(\mathrm{gj}) \pm$} & 0.16 & 1.53 & 0.091 & 0.64 & 0.08 \\
\hline
\end{tabular}

*, ** Significant at 5 and 1 per cent probability levels, respectively

Table 3: Estimation of specific combining ability for different characters in cucumber

\begin{tabular}{|c|c|c|c|c|c|c|c|c|c|}
\hline $\begin{array}{l}\text { Sr. } \\
\text { No. }\end{array}$ & Hybrids & \begin{tabular}{|c|}
$\begin{array}{c}\text { Days to first male } \\
\text { flower } \\
\text { appearance }\end{array}$ \\
\end{tabular} & $\begin{array}{l}\text { Days to first } \\
\text { female flower } \\
\text { appearance }\end{array}$ & \begin{tabular}{|c|}
$\begin{array}{c}\text { Nodal position } \\
\text { of first female } \\
\text { flower }\end{array}$ \\
\end{tabular} & $\begin{array}{c}\text { Days to } \\
\text { first } \\
\text { picking }\end{array}$ & $\begin{array}{c}\text { Fruit } \\
\text { length } \\
(\mathrm{cm})\end{array}$ & $\begin{array}{c}\text { Fruit } \\
\text { diameter } \\
(\mathrm{cm})\end{array}$ & \begin{tabular}{|c|} 
Average \\
fruit weight \\
$(\mathrm{g})$
\end{tabular} & $\begin{array}{l}\text { Number of } \\
\text { marketable } \\
\text { fruits vine }^{-1}\end{array}$ \\
\hline 1 & $\begin{array}{l}\text { Sheetal } x \\
\text { AAUC-2 }\end{array}$ & 0.479 & 1.042 & 0.958 & -1.396 & 0.730 & -0.084 & -1.977 & $-1.125^{*}$ \\
\hline 2 & Sheetal x DC-2 & $2.354 *$ & 0.417 & 0.958 & 1.229 & 0.088 & 0.208 & 0.310 & $-1.238^{* *}$ \\
\hline 3 & $\begin{array}{l}\text { Sheetal } \mathrm{x} \\
\text { AAUC-1 }\end{array}$ & $2.854 *$ & 1.042 & $1.958 * *$ & 0.604 & -0.879 & 0.231 & $-25.602 *$ & -0.750 \\
\hline 4 & $\begin{array}{c}\text { Sheetal x VRC- } \\
19\end{array}$ & 0.104 & 0.917 & -0.417 & 2.104 & $-1.153^{*}$ & 0.051 & 10.060 & $1.500^{* *}$ \\
\hline 5 & $\begin{array}{c}\text { Sheetal x DARL- } \\
103\end{array}$ & -1.271 & 0.542 & $-1.667 * *$ & 0.854 & $1.618 * *$ & -0.319 & 15.623 & 0.450 \\
\hline 6 & $\begin{array}{c}\text { Sheetal x Fansu } \\
\text { local }\end{array}$ & $-4.521 * *$ & $-3.958 * *$ & $-1.792 * *$ & $-3.396 * *$ & -0.404 & -0.087 & 1.585 & $1.163^{*}$ \\
\hline 7 & $\begin{array}{c}\text { Shubhangi } x \\
\text { AAUC-2 }\end{array}$ & $2.313^{*}$ & $3.792 * *$ & 0.125 & $5.271 * *$ & -0.306 & 0.172 & -14.519 & -0.033 \\
\hline 8 & Shubhangi x DC- & $-2.813^{*}$ & $-2.333^{*}$ & 0.125 & -1.104 & 0.402 & 0.435 & -9.731 & $1.154 *$ \\
\hline 9 & $\begin{array}{c}\text { Shubhangi x } \\
\text { AAUC-1 }\end{array}$ & -1.313 & -1.708 & $-1.375^{*}$ & -0.729 & -0.535 & 0.027 & 18.306 & $2.092 * *$ \\
\hline 10 & $\begin{array}{c}\text { Shubhangi x } \\
\text { VRC-19 }\end{array}$ & 1.438 & 0.667 & -0.750 & -2.229 & $1.396^{*}$ & -0.302 & -0.031 & $-1.358 * *$ \\
\hline 11 & $\begin{array}{c}\text { Shubhangi } x \\
\text { DARL-103 }\end{array}$ & $2.563^{*}$ & -0.208 & 1.000 & 0.021 & -0.958 & -0.283 & -1.919 & 0.092 \\
\hline 12 & $\begin{array}{c}\text { Shubhangi } x \\
\text { Fansu local }\end{array}$ & $-2.188^{*}$ & -0.208 & 0.875 & -1.229 & 0.000 & -0.050 & 7.894 & $-1.946 * *$ \\
\hline 13 & $\begin{array}{l}\text { Himangi } x \\
\text { AAUC-2 }\end{array}$ & -0.104 & $-2.375^{*}$ & -0.875 & $-2.563^{*}$ & -1.013 & -0.346 & 8.323 & 0.025 \\
\hline 14 & Himangi x DC-2 & $-5.229 * *$ & -1.000 & 0.625 & -0.938 & -0.414 & -0.303 & 11.510 & $0.913 *$ \\
\hline
\end{tabular}




\begin{tabular}{|c|c|c|c|c|c|c|c|c|c|}
\hline 15 & $\begin{array}{l}\text { Himangi } x \\
\text { AAUC-1 }\end{array}$ & $-3.229 * *$ & -0.875 & -0.375 & -1.563 & $1.138^{*}$ & -0.451 & 18.348 & $1.150 *$ \\
\hline 16 & $\begin{array}{c}\text { Himangi x VRC- } \\
19\end{array}$ & 0.021 & $-2.000 *$ & -0.250 & 1.438 & -0.360 & 0.129 & -5.040 & $-1.750 * *$ \\
\hline 17 & $\begin{array}{l}\text { Himangi } x \\
\text { DARL-103 }\end{array}$ & $2.646^{*}$ & $3.625 * *$ & 0.500 & 0.188 & 0.526 & $0.989 * *$ & $-34.077 * *$ & $1.100 *$ \\
\hline 18 & $\begin{array}{c}\text { Himangi x Fansu } \\
\text { local }\end{array}$ & $5.896 * *$ & $2.625^{*}$ & 0.375 & $3.438 * *$ & 0.123 & -0.018 & 0.935 & $-1.438 * *$ \\
\hline 19 & $\begin{array}{l}\text { Punakhira } \mathrm{x} \\
\text { AAUC-2 }\end{array}$ & $-2.688^{*}$ & $-2.458^{*}$ & -0.208 & -1.313 & 0.590 & 0.258 & 8.173 & $1.133 *$ \\
\hline 20 & \begin{tabular}{|c|} 
Punakhira $\mathrm{x}$ DC- \\
2
\end{tabular} & $5.688 * *$ & $2.917 * *$ & $-1.708 * *$ & 0.813 & -0.077 & -0.340 & -2.090 & -0.829 \\
\hline 21 & $\begin{array}{l}\text { Punakhira } \mathrm{x} \\
\text { AAUC-1 }\end{array}$ & 1.688 & 1.542 & -0.208 & 1.688 & 0.276 & 0.193 & -11.052 & $-2.492 * *$ \\
\hline 22 & $\begin{array}{l}\text { Punakhira } x \\
\text { VRC-19 }\end{array}$ & -1.563 & 0.417 & $1.417 *$ & -1.313 & 0.117 & 0.122 & -4.990 & $1.608 * *$ \\
\hline 23 & $\begin{array}{l}\text { Punakhira } x \\
\text { DARL-103 }\end{array}$ & $-3.938 * *$ & $-3.958 * *$ & 0.167 & -1.063 & $-1.187 *$ & -0.387 & 20.373 & $-1.642 * *$ \\
\hline 24 & $\begin{array}{l}\text { Punakhira } \mathrm{x} \\
\text { Fansu local } \\
\end{array}$ & 0.813 & 1.542 & 0.542 & 1.188 & 0.281 & 0.155 & -10.415 & $2.221 * *$ \\
\hline \multicolumn{2}{|c|}{$\begin{array}{c}\text { No. of crosses showed } \\
\text { (-ve) significant sca } \\
\text { effects }\end{array}$} & & 06 & 04 & 02 & 07 & 01 & - & 10 \\
\hline & $\mathrm{SE} \pm$ & & 1.22 & 0.73 & 1.53 & 1.35 & 0.35 & 13.02 & 0.56 \\
\hline
\end{tabular}

Table 3: cont.

\begin{tabular}{|c|c|c|c|c|c|c|}
\hline Sr. No. & Hybrids & $\begin{array}{c}\text { Marketable yield vine } \\
\mathbf{1}(\mathbf{k g})\end{array}$ & $\begin{array}{c}\text { Harvest } \\
\text { duration }\end{array}$ & $\begin{array}{c}\text { Number of primary } \\
\text { branches plant }^{-1}\end{array}$ & \begin{tabular}{|c|}
$\begin{array}{c}\text { Vine length } \\
(\mathrm{m})\end{array}$ \\
\end{tabular} & $\begin{array}{c}\text { Total soluble solids } \\
\left({ }^{0} \mathrm{Brix}\right)\end{array}$ \\
\hline 1 & Sheetal x AAUC-2 & 0.134 & 0.458 & -0.027 & -0.116 & $-0.250 *$ \\
\hline 2 & Sheetal x DC-2 & $-0.550^{*}$ & -2.167 & 0.148 & 0.217 & 0.238 \\
\hline 3 & Sheetal x AAUC-1 & $-0.745 * *$ & 0.083 & -0.015 & -0.221 & $0.562 * *$ \\
\hline 4 & Sheetal x VRC-19 & 0.257 & -2.042 & -0.002 & -0.308 & $0.350 * *$ \\
\hline 5 & Sheetal x DARL-103 & -0.038 & 0.958 & -0.002 & 0.259 & -0.012 \\
\hline 6 & Sheetal x Fansu local & $0.942 * *$ & 2.708 & -0.102 & 0.169 & $-0.888 * *$ \\
\hline 7 & Shubhangi x AAUC-2 & -0.079 & 2.875 & $0.281 *$ & 0.301 & 0.042 \\
\hline 8 & Shubhangi x DC-2 & $0.532 *$ & 2.750 & -0.044 & -0.037 & $-0.521 * *$ \\
\hline 9 & Shubhangi x AAUC-1 & 0.407 & -2.000 & -0.156 & 0.106 & $-0.546 * *$ \\
\hline 10 & Shubhangi x VRC-19 & $-0.517 *$ & -1.625 & -0.094 & -0.272 & $0.492 * *$ \\
\hline 11 & Shubhangi x DARL-103 & 0.183 & 1.375 & 0.006 & -0.204 & 0.229 \\
\hline 12 & Shubhangi x Fansu local & $-0.527 *$ & -3.375 & 0.006 & 0.106 & $0.304^{*}$ \\
\hline 13 & Himangi x AAUC-2 & -0.257 & $-4.875^{*}$ & -0.185 & 0.669 & -0.225 \\
\hline 14 & Himangi x DC-2 & 0.385 & 1.500 & -0.110 & -0.148 & $0.463 * *$ \\
\hline 15 & Himangi x AAUC-1 & 0.420 & 3.750 & 0.177 & -0.416 & 0.138 \\
\hline 16 & Himangi x VRC-19 & -0.224 & $4.625^{*}$ & 0.240 & 0.367 & $-0.675 * *$ \\
\hline 17 & Himangi x DARL-103 & 0.341 & -2.375 & 0.040 & -0.226 & 0.113 \\
\hline 18 & Himangi x Fansu local & $-0.664 * *$ & -2.625 & -0.160 & -0.246 & 0.187 \\
\hline 19 & Punakhira x AAUC-2 & 0.202 & 1.542 & -0.069 & -0.854 & $0.433 * *$ \\
\hline 20 & Punakhira x DC-2 & -0.367 & -2.083 & 0.006 & -0.032 & -0.179 \\
\hline 21 & Punakhira x AAUC-1 & -0.082 & -1.833 & -0.006 & 0.531 & -0.154 \\
\hline 22 & Punakhira x VRC-19 & $0.484 *$ & -0.958 & -0.144 & 0.213 & -0.167 \\
\hline 23 & Punakhira x DARL-103 & $-0.486^{*}$ & 0.042 & -0.044 & 0.171 & $-0.329 *$ \\
\hline 24 & Punakhira x Fansu local & 0.249 & 3.292 & 0.256 & -0.029 & $0.396 * *$ \\
\hline \multicolumn{2}{|c|}{$\begin{array}{c}\text { No. of crosses showed (-ve) significant } \\
\text { sca effects }\end{array}$} & 03 & 01 & 01 & - & 07 \\
\hline & $\mathrm{SE} \pm$ & 0.28 & 2.80 & 0.16 & 0.56 & 0.15 \\
\hline
\end{tabular}

\section{Days to first male flower appearance}

Earliness is desirable character, the exhibited significant negative or positive GCA effects were designated as good or poor general combiners, respectively. The remaining males exhibited non-significant GCA effects were assigned as average general combiners for early male flower appearance.

For days to first male flower appearance the estimates of GCA effects revealed that the single female parent Himangi (4.396) and three male parents viz, DC-2(-2.188), AAUC-1 (2.688) and DARL-103 (-0.063) had significant negative GCA effects.
The hybrids having highest significant negative sca effects in order were Himangi $\times$ DC-2(-5.229), Sheetal x Fansu local (4.521) and Puna khira $x$ DARL-103 (-3.938). The cross combinations exhibited significant negative sca effects were designated as good specific combiners. Hence, they were considered as good cross combinations for exploiting earliness in male flowering.

\section{Days to first female flower appearance}

In days to first female flower appearance the estimates of gca effects revealed that the two female parent viz. Himangi (1.875) and Shubhangi (-0.042) and three male parents viz. 
AAUC-1-(1.708), DARL-103(-1.208) and DC-2(-1.083) had significant negative gca effects.),Pati et al. (2015) ${ }^{[13]}$, Kumar and Kumar (2017) ${ }^{[5]}$ and Naik et al. (2018) ${ }^{[10]}$ had revealed significant gca effects for this trait.

The estimates of sca effects revealed that significant negative sca effects in order were Sheetal x Fansu local and Puna khira $x$ DARL-103 exihibited (-3.958) same sca effects. The cross combinations in $F_{1}$ exhibited significant negative sca effects were designated as good specific combiners. These cross combinations were exploiting earliness and used in crop improvement. Kaur and Dhall (2017) ${ }^{[3]}$ and Nimitha et al. (2017) ${ }^{[11]}$ reported highly significant sca effects for above trait.

\section{Nodal position of first female flower}

In nodal position of first female flower the estimates of gca effects revealed that the two female parent Puna khira (0.417), Shubhangi (-0.250) and three male parents viz, DARL-103 (-1.083), VRC-19 (-0.333) and DC-2 (-0.208) had exhibited significant negative gca effects Thus, negative estimates of gca effects indicated the parents are good general combiners for node bearing of first female flower. Kumar and Kumar (2017) ${ }^{[5]}$ recorded highly significant gca effects for nodal position of female.

The estimates of sca effects revealed that four hybrids recorded significant negative sca effects. The hybrids having highest significant negative sca effects in order were Sheetal $\times$ Fansu local (-1.792), and Puna khira x DC-2 (-1.708). The cross combinations were designated as good specific combiners which bearing first female flower earlier. Pati et al. (2015) ${ }^{[13]}$, Tiwari and Singh (2016) ${ }^{[21]}$ and Kaur and Dhall (2017) ${ }^{[3]}$. The cross combinations were designated as good specific combiners which bearing first female flower earlier.

\section{Days to first picking}

An examination of gca estimates revealed that the three female parents viz., Himangi (-1.813), Sheetal (-0.979), Shubhangi (-0.146) and among male parents DC-2 (-1.396), AAUC-2 (-0.271) and DARL-103 (-0.021) recorded significant negative gca effects.In overall result in the female parent Himangi is good general combiner in all season and on pooled basis in early picking. Singh and Ram (2016) ${ }^{[19]}$ and Bhutia et al. (2017) [2] also reported similar finding in cucumber.

The estimates of sca effects revealed that two hybrids Sheetal $\times$ Fansu local (-3.396) and Himangi x AAUC-2 (-2.563) were found good specified combination designated as good specific combiners in early picking character These results are in agreement with Reddy et al. (2014) ${ }^{[15]}$.

\section{Fruit length $(\mathrm{cm})$}

The estimates of gca effects revealed that only one female parent Shubhangi (0.263) exhibited highly significant positive gca effects while in only one male parent DARL-103 (0.336) showed significant positive gca effects. This indicated that these parents were good general combiners for this character. Naik et al. (2018) ${ }^{[10]}$ and Pati et al. (2015) ${ }^{[13]}$ explain the positive gca effects for this trait.

A study of estimates for the sca effect revealed that three hybrids viz., sheetal x DARL-103 (1.618), Shubhangi x VRC - 19 (1.396) and Himangi x AAUC-1 (1.138) highly significant positive sca effects this indicates that they are good specific combiners for this trait. Similar results were reported by earlier workers Mule et al. (2012), Xian and Ying (2012), Kaur and Dhall (2017) and Malav and Verma (2018) $[9,23,3,7]$.

\section{Fruit diameter $(\mathbf{c m})$}

For fruit diameterthe estimates of gca effects revealed that only one female parent Punakhira (0.548) exhibited highly significant positive gca effects. These parents were good general combiners for said trait. As regard to sca effects only one hybrid Himangi x DARL-103 exhibited highly (0.989) significant positive sca effects. Malav and Verma (2018) ${ }^{[7]}$ had reported highly significant gca and sca effects for this trait.

\section{Average fruit weight (g)}

Theaverage fruit weight estimates of gca and sca effects wasnon significant indicating that they are poor specific combiners for this trait.

\section{Number of marketable fruits per vine}

An examination of gca estimates revealed that among these two female parents Sheetal (2.700) and Punakhira (1.191) as well as three male parents Fansu local (1.604), VRC-19 (0.817) and AAUC-2(0.692) exhibited highest sca effects These parents were exhibits good general combining ability. These results were in similar line with Pati et al. (2015) [13] and Reddy et al. (2014) ${ }^{[15]}$.

A perusal of estimates of sca effects of hybrids revealed that ten hybrids showed significant positive sca effects, among these Puna khira x Fansu local (2.221) and Shubhnagi $\mathrm{x}$ AAUC-1(2.092) recorded highest significant positive sca effects.Bhutia et al. (2017) ${ }^{[2]}$ and Naik et al. (2018) [10] obtained similar findings for this trait.

\section{Marketable yield per vine (kg)}

The perusal of estimates of gca effects revealed that two female parents viz., Sheetal (0.572), Puna khira (0.235) and two male parents Fansu local (0.596) and VRC-19(0.376) exhibited significant positive gca effect These showed that these parents were good general combiner for the said trait. Ahammed et al. (2018) ${ }^{[1]}$ exhibits similar finding for this fruit.

With regards to sca effect three hybrids towards significant positive direction for this trait. The best three hybrids with respect to marketable yield vine ${ }^{-1}$ based on significant positive sca were viz., Sheetal x Fansu local (0.942), Shubhangi x DC2 (0.532), Puna khira x VRC-19 (0.484). Kumar and Kumar (2017) ${ }^{[5]}$ and Moradipour et al. (2017) ${ }^{[8]}$ found similar results for positive marketable yield in cucumber.

\section{Harvest duration}

An examination of gca estimates all male and female parents had non significant gca effects.

A perusal of estimates of sca effects of hybrids revealed that onlyone Himangi x VRC-19 (4.625), hybrid recorded significant positive sca effects registered significant highest positive effects which are good specific combiner for longest harvest duration.

\section{Number of primary branches per plant}

An examination of gca estimates revealed that female parent Sheetal (0.502) and Punakhira (0.544) recorded significant positive gca effects.

A perusal of estimates of sca effects of hybrids revealed that two hybrids Shubhnagi x AAUC-2(0.281) and Punakhira $\mathrm{x}$ Fansu local (0.283) recorded significant positive sca effects. Similar findings were obtained by Prasad and Singh (1992) ${ }^{[14]}$. 


\section{Vine length (m)}

The persual of estimates of gca and sca effects indicated that all these parents and hybrids exhibitsn on significant gca and sca effects respective.

\section{Total soluble solids ( $\left.{ }^{0} \mathrm{Brix}\right)$}

Based on estimates of general combining ability effects among female two parents Sheetal (0.263) and Himangi (0.138) were observed to be the good general combiners while three male parents viz., AAUC-2(0.317), DARL-103(0.079) and Fansu local (0.054).The parent had highest gca effects exhibits good general combining ability. Similar findings obtained by Li. et. al. (2005) ${ }^{[6]}$ and Reena Kumari et al. (2017) ${ }^{[16]}$.

In regards to sca effects seven hybrids showed positive significant sca effects. Among these the hybrids Sheetal $\mathrm{x}$ AAUC-1 (0.562) displayed highly significant positive sca effects followed by Shubhangi x VRC-19 (0.492) and Himangi x DC-2(0.463). This indicated that these were good general combiner for total soluble solids. Nimitha et al. (2017) [11] and Malav and Verma (2018) [7] revealed the similar results for estimation of highest sca effects for this trait.
The best performing hybrids were found to be good specific combinations as evident from its significant positive sca effects. Expliotation for these traits might also be possible in $\mathrm{H} \times \mathrm{L}$ and $\mathrm{L} \times \mathrm{L}$ cross combinations.

\section{Gene action}

A perusal of the data indicated that (Table 4) the estimates of $\sigma^{2}$ SCA were higher in magnitude as compared to $\sigma^{2}$ GCA (average) both in females and males for the traits under study viz., days to first male flower appearance, days to first female flower appearance, nodal position of first female flower, days to first picking, fruit length and diameter, average fruit weight, marketable yield vine ${ }^{-1}$, harvest duration and total soluble solids. In all the traits studied, the SCA variances were higher than GCA values, indicated the role of nonadditive gene action. While lower magnitude of SCA showed additive gene action. Components of dominance variance $\left(\sigma^{2} \mathrm{D}\right)$ were also higher than the additive components $\left(\sigma^{2} \mathrm{~A}\right)$ indicating the role of non-additive gene action. For the five traits viz., days to first male flower appearance, days to first female flower appearance, nodal position of first female flower, fruit length, average fruit weight and total soluble solids.

Table 4: Estimation of random effect of GCA and SCA variances, additive and dominance variance components, heritability and genetic advance in cucumber

\begin{tabular}{|c|c|c|c|c|c|c|c|c|c|c|}
\hline $\begin{array}{l}\text { Sr. } \\
\text { No. }\end{array}$ & Characters & \begin{tabular}{|c}
$\sigma^{2}$ GCA of \\
Females
\end{tabular} & $\begin{array}{c}\sigma^{2} \text { GCA of } \\
\text { Males }\end{array}$ & $\begin{array}{c}\sigma^{2} \text { GCA } \\
\text { (Average) }\end{array}$ & $\begin{array}{c}\sigma^{2} \text { SCA of } \\
\text { female } x \text { male }\end{array}$ & $\sigma^{2} \mathbf{A}$ & $\sigma^{2} \mathbf{D}$ & $\begin{array}{l}\sigma^{2} \mathbf{A} / \\
\sigma^{2} D\end{array}$ & $\begin{array}{c}\mathbf{h}^{2} \\
\text { in } \%\end{array}$ & $\begin{array}{c}\text { Genetic } \\
\text { Advance }\end{array}$ \\
\hline 1 & Days to first male flower appearance & 6.63 & 0.93 & 4.354 & 12.56 & 8.70 & 12.56 & 0.69 & 38.92 & 3.79 \\
\hline 2 & Days to first female flower appearance & 0.94 & 0.68 & 0.844 & 6.36 & 1.68 & 6.36 & 0.26 & 18.85 & 1.16 \\
\hline 3 & Nodal position of first female flower & -0.10 & 0.13 & -0.0078 & 1.22 & -0.015 & 1.22 & -0.012 & -1.01 & -0.02 \\
\hline 4 & Days to first picking & 3.30 & 0.83 & 2.3122 & 4.58 & 4.62 & 4.58 & 1.009 & 43.57 & 2.92 \\
\hline 5 & Fruit length $(\mathrm{cm})$ & -0.10 & -0.11 & -0.1103 & 0.64 & -0.22 & 0.64 & -0.34 & -31.89 & -0.54 \\
\hline 6 & Fruit diameter $(\mathrm{cm})$ & 0.17 & -0.00 & 0.099 & 0.08 & 0.19 & 0.08 & 2.26 & 55.20 & 0.68 \\
\hline 7 & Average fruit weight $(\mathrm{g})$ & -14.87 & 51.77 & 11.78 & 176.92 & 23.56 & 176.92 & 0.13 & 7.79 & 2.79 \\
\hline 8 & Number of marketable fruits vine ${ }^{-1}$ & 5.02 & 0.88 & 3.371 & 2.83 & 6.74 & 2.83 & 2.37 & 69.02 & 4.44 \\
\hline 9 & Marketable yield vine $^{-1}(\mathrm{~kg})$ & 0.186 & 0.107 & 0.154 & 0.255 & 0.309 & 0.255 & 1.212 & 50.34 & 0.81 \\
\hline 10 & Harvest duration & 2.35 & 3.63 & 2.863 & 5.21 & 5.72 & 5.21 & 1.09 & 36.58 & 2.98 \\
\hline 11 & Number of primary branches plant ${ }^{-1}$ & 0.369 & 0.0128 & 0.228 & 0.01 & 0.453 & 0.01 & 45.28 & 94.40 & 1.34 \\
\hline 12 & Vine length $(\mathrm{m})$ & 0.033 & -0.017 & 0.0131 & -0.64 & 0.026 & \begin{tabular}{|l|}
-0.647 \\
\end{tabular} & -0.0406 & 13.59 & 0.12 \\
\hline 13 & Total soluble solids $\left({ }^{0}\right.$ Brix $)$. & 0.035 & -0.02 & 0.0113 & 0.226 & 0.0226 & 0.2276 & 0.0993 & 8.54 & 0.09 \\
\hline
\end{tabular}

\section{Heritability}

The effectiveness of selection for a trait depends on relative importance of genetic and non-genetic factors in the expression of phonotypic difference among genotypes in population, a concept referred to as heritability. The heritability has major impact on the methods chosen for population improvement, in breeding and other aspect of single plant selection may be effective for the character with low (5 to10\%), medium (10 to $30 \%$ ), high (30 to 60\%) and very high (above 60\%) heritability (Robinson, 1966) ${ }^{[18]}$.

The heritability estimates were made from different variance components (Table 4)obtained for kharif 2017 very high heritability obtained for number of primary branches plant ${ }^{-}$ ${ }^{1}(94.40 \%)$, number of marketable fruits vine ${ }^{-1}(69.02 \%)$. High heritability observed for marketable yield vine ${ }^{-1}(50.34 \%)$ similar results obtained by $\mathrm{Gu}$-Xing et al. (2004) for this character. The fruit diameter $(55.20 \%)$,days to first picking $(43.57 \%)$ and days to first male flower appearance $(38.92 \%)$, similar findings obtained by Uddin et al. (2009) ${ }^{[22]}$ for all these characters. The harvest duration $(36.58 \%)$ recorded high heritability which had utility in prolongs the duration of harvesting in cross combination.

\section{References}

1. Ahammed S, Hossain M, Zakaria M, Ahmed MB, Mian MAK. Combining ability and gene action in cucumber (Cucumis sativus L.). J. Agric. Studies. 2018; 6(2):145159.

2. Bhutia TL, Munshi AD, Behera TK, Sureja AK, Lal SK. Combining ability for yield and yield related traits and its relationship with gene action in cucumber. Indian J. Hort. 2017; 74(1):51-55.

3. Kaur K, Dhall RK. Heterosis and combining ability for yield and yield Attributes in cucumber (Cucumis sativus L.) Sabrao J. Breed. Genet. 2017; 49(1):94-103.

4. Kempthorne O. An introduction to Genetical Statistics. John Wiley and Sons, New York, London, 1957, 458$471 \mathrm{p}$.

5. Kumar R, Kumar S. Usefulness of combining ability and gene action studies for parthenocarpic gynoecious hybrid development in cucumber. J. Hill Agric. 2017; 8(2):158165.

6. Li LL, Xu Q, Chen X. Analysis of combining ability on quality characters of pickling cucumber. China Vegetables. 2005; 1:13-15. 
7. Malav N, Verma S. Heterosis in cucumber (Cucumis sativus L.). Int. J. Chem. Stud. 2018; 6(3):1538-1540.

8. Moradipour F, Olfati J, Hamidoghli AY, Sabouri A, Zahedi B. General and specific combining ability and heterosis for yield in cucumber fresh market lines. Inter. J. Vege. Sci. 2017; 23(4):285-293.

9. Mule PN, Khandelwal V, Lodam VA, Shinde DA, Patil PP, Patil AB. Heterosis and combining ability in cucumber (Cucumis sativus L.) Madras Agric. J. 2012; 99(7-9):420-423.

10. Naik P, Nagarajappa AR, Srinivasa V, Gangaprasad S, Shridhar PH. Combining ability studies in cucumber (Cucumis sativus L.). Int. J. Pure App. Bio Sci. 2018; 6(2):1389-1393.

11. Nimitha K, Acharya RR, Maibam U. Analysis of combining ability in cucumber (Cucumis sativus L.). Inter. J. Agric. and Envir. Res. 2017; 3(3):3358-3364.

12. Panse VG, Sukhatme PV. Statistical methods for agricultural workers, Indian Council of Agricultural Research, New Delhi, 1985, 381p.

13. Pati K, Munshi AD, Behera TK, Kumar R, Karmakar P. Estimating combining ability for yield and yield contributing traits in cucumber. Indian J. Hort. 2015; 72(1):49-53.

14. Prasad KVSR, Singh DP. Combining ability through line $\mathrm{x}$ tester analysis in cucumber (Cucumis sativus L.). Indian J. Hort. 1992; 49(4):358-362.

15. Reddy KAN, Munshi AD, Behera TK, Sureja AK, Sharma RK. Studies on combining ability in cucumber. Indian J. Hort. 2014; 71(3):349-353.

16. Reena Kumari, Ramesh Kumar, Sandeep Kumar. Combining ability and heterosis for TSS and seed traits in cucumber (Cucumis sativus L). Veg. Sci. 2017; 44(1):1217.

17. Renner SS, Schaefer H, Kocyan A. Phylogenetics of Cucumis (Cucurbitaceae): cucumber (C. sativus) belongs in an Asian/Australian clade far from melon (C. melo). BMC Evolutionary Biology. 2007; 7:58.

18. Robinson HF. Quantitative genetics in relation to breeding on central of Mendalism. Indian J. Genet. 1966; 26A:171-187.

19. Singh A, Ram HH. Estimates of stability parameters for yield and its components in cucumber (Cucumis sativus L.). Veg. Sci. 2016; 39(1):31-34.

20. Tasdighi M, Baker LR. Combining ability for femaleness and yield in single and 3-way crosses of pickling cucumbers intended for once-over harvest. Euphytica. 1981; 30(1):183-192.

21. Tiwari R, Singh DK. Study of heterosis and combining ability for earliness and vegetative traits in cucumber (Cucumis sativus L.) Journal of Applied and Natural Science. 2016; 8(2):999-1005.

22. Uddin NM, Hossain MM, Rahman M, Ahmad MS, Quamruzzaman AKM. Combining ability and gene action in cucumber (Cucumis sativus L.) SAARC J. Agric. 2009; 7(1):64-72.

23. Xian ZJ, Ying L. Analysis on combining ability and heritability of main fruit characters of cucumber parents. Acta Agriculturae Jiangxi. 2012; 24(1):12-14. 